\title{
The Emerging Role of Platelet-Activating Factor on the Regulation of Embryonic Ploidy via Apoptosis
}

\author{
Sparsha Saxena, Harrison Lapin, Renee J Chosed and William E Roudebush* \\ Department of Biomedical Sciences, University of South Carolina School of Medicine Greenville, USA
}

\begin{abstract}
Sea Urchins have been one of the principal animals of choice to study the mechanism's regarding fertilization and embryo development. A key influencer of events during embryo development is apoptosis, which can assist in modifying chromosome normality. Platelet-activating factor (PAF), a potent phospholipid, is produced by spermatozoa and early embryos and is required for fertilization and development. PAF has profound effects on fertilization and early embryo development including, but not limited to, apoptosis. This review will look at the significant relationship between PAF and apoptosis during early embryo development with emphasis in the sea urchin and to suggest next steps for study.
\end{abstract}

KEYWORDS: Platelet-activating factor; Apoptosis: Embryogenesis

ABBREVIATIONS: PAF: Platelet-Activating Factor; IVF: In Vitro Fertilization

\section{INTRODUCTION}

In 1972, Benveniste et al. [1] used the term Platelet Activating Factor (PAF) to describe a substance that leukocytes released upon stimulation that led to platelet aggregation as well as histamine release in rabbits [2]. While the efforts leading to PAF's discovery were aimed at elucidating the mechanism behind leukocytedependent histamine release, it is now known that PAF plays a critical role in a diverse set of biological pathways.

At the time, PAF was thought to solely function as a lipid mediator, which initiates movement of leukocytes to begin an immune reaction [3]. Further structural research, however, revealed that PAF is a phospholipid that uniquely was able to function in signaling, specifically in a juxtacrine, paracrine, autocrine, and endocrine manner [2]. Consequently, throughout the years research has shown that PAF mediates a variety of cellular interactions beyond inflammation, such as long-term potentiation, apoptosis, and reproduction [4-6]. It is also implicated in a host of disease states that begin at the cellular level such as cancer, cardiovascular disease, and renal disease [7-9].

Of growing interest, however, is PAF's relevance in early preimplantation embryo development and subsequent implantation, and consequently, embryo viability and a successful pregnancy. Embryos produce high levels of PAF, and the amount of embryo derived PAF increases at variable rates throughout development, suggesting a critical role in embryogenesis [10]. This review revisits research establishing PAF's role during embryo development as well as explores the emerging studies establishing a connection between PAF and apoptosis pathways as a means to promote development and implement implantation.
Quick Response Code:

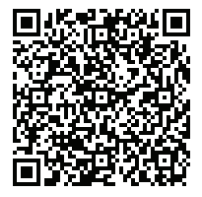

Address for correspondence: William E Roudebush, Department of Biomedical Sciences, University of South Carolina School of Medicine Greenville, USA

Received: January 22, $2021 \quad$ Published: February 04, 2021

How to cite this article: Sparsha S, Harrison L, Renee J C, William ER. The Emerging Role of Platelet-Activating Factor on the Regulation of Embryonic Ploidy via Apoptosis. 2021- 3(1) OAJBS.ID.000252. DOI: $10.38125 /$ OAJBS.000252 


\section{DISCUSSION}

\section{Mammalian PAF Studies}

Our understanding of PAF's role in embryo development has been expanded over the years. Preimplantation stage embryos in a variety of species (human, mouse, sheep, rabbit and pig) produce and release PAF [11-14]. The importance of PAF's role in embryonic development was documented when it was shown that PAF-antibodies inhibited embryonic development [15] and that PAF-antagonists inhibited implantation [16-17] providing further evidence on the presence and requirement of embryoderived PAF during the pre-, peri- and implantation periods. Roberts et al. [18] showed that mouse preimplantation embryos in the presence of exogenous PAF have enhanced developmental rates higher implantation rates upon transfer to synchronized recipients [19] and larger litter sizes [20]. It was hypothesized that these results may be due to embryo-derived PAF stimulation of embryonic metabolism [21]. Additionally, investigations provided evidence that PAF directly influences the oxidative metabolism of glucose and lactate in the mouse preimplantation embryo [22]. Further evidence was provided that showed enhanced embryo development occurred in rabbit oocytes that were fertilized in vitro with PAF treated spermatozoa [23].

\section{PAF Mechanism Studies}

PAF released from the embryo can cause changes in maternal physiology. These changes include, but are not limited to platelet activation, changes in oviductal, endometrial and maternal immune function, and has even been shown to act as an autocrine molecule that acts as a survival factor for the early embryo [24]. Embryo survivability was limited in an experiment that blocked PAF's autocrine action [24]. This could be because embryonic-PAF acts as an autocrine stimulator of embryo development [25]. The action(s) of this embryo derived PAF on development were demonstrated by an experiment that used PAF-antibodies or PAF-antagonists that acted to block the frequency of complete embryonic development [26].

In humans, PAF has been shown to act through paracrine pathways to control embryo transport through the oviduct [27]. PAF production by human embryos has been correlated with pregnancy potential, the ability for the embryos to successfully implant $[28,29]$ In vitro, supplementation of culture media with PAF has shown to improve embryo development, although this mechanism is not well understood [24]. This has led to further research on the mechanisms of action of PAF because of the advances it could provide to women with fertility problems. Treatment of mouse embryos resulted in a significant improvement of delivery rates and birth weights [30]. Improved rabbit blastocyst development after in vitro fertilization with PAF treated spermatozoa has also been reported [23]. In other cell types, PAF receptor and its believed mechanism of action has been reported as a receptor-mediated process. PAF's action in the embryo is also a receptor-mediated since different PAF-antagonists competitively inhibit its action [26].

\section{Sea Urchins Model to Study PAF Molecular Mechanisms}

Sea Urchins have been one of the animals of choice to study the PAF mechanism regarding fertilization and development. Sea urchins are a class of marine invertebrates that possess many genetic similarities to humans [31]. They are estimated to have over 23,000 genes similar to vertebrae's and have long been used as models in developmental biology studies [31,32]. Recently, sea urchins have been used more frequently to study the PAF molecule and its role in embryo development due to the ease of using in the lab and the speed at which fertilization is completed [33]. It is important to understand the PAF molecular mechanisms that modulate fertilization and development in order to potentially enhance in vitro fertilization (IVF) remain outcomes.

Using the Sea urchins as a model, PAF was shown to be a molecule that increases sperm motility and fertilizing capability [32]. The collective data provides further evidence that PAF's effect on development is receptor-mediated and may involve the inositol triphosphate system. PAF binding to its receptor on the embryo results in increased intracellular calcium levels which enhance sperm capacitation and cell motility leading to increased fertilization [34]. This helped establish the mechanism by which PAF affects fertility, but not development. It was shown that exogenous PAF induced advanced stages of embryo development [33]. However, the mechanism is still not fully understood. One such hypothesis is the relationship between PAF and apoptosis in embryos and figuring out the mechanism associated with this process could lead to a valuable breakthrough in IVF fertility rates [35].

\section{Apoptosis and Embryogenesis}

Even though PAF works through a range of pathways to induce different outcomes during embryonic development, it is postulated that PAF promotes implantation. In other cell systems, apoptosis appears to be one mechanism by which PAF functions, either through activation or inhibition of apoptosis.

For example, in exploring the nervous system in mice, deleting the PAF receptor led to high levels of circulating PAF which triggered caspase 3/7 activity and neuronal death [36]. In this system, PAF's receptor may prevent apoptosis. In addition, when both rabbit and human corneal cells were exposed to ultraviolet radiation, $\mathrm{PAF}$, cytochrome $\mathrm{C}$, and caspase levels increased only in ultraviolet damaged cells. When PAF was exogenously delivered to corneal cells, they also underwent apoptosis [37].

To study apoptosis in embryogenesis, however, sea urchin experiments provide insight on the influence of PAF. Apoptosis may not occur during cleavage phase [38-40]. Low rates of apoptosis have been observed during the blastula and gastrula phase [38]. This timing when programmed cell death occurs is not altered even in the presence of chemical insults [38]. Prior studies sought to apply a variety of chemicals (for example, staurosporine, etopside and emetine) to induce apoptosis on the sea urchin and monitor the consequences. Inappropriately turning on apoptosis in the sea urchin through genetically removing cell cycle checkpoint genes showed that once the embryo entered the gastrula they were no longer viable.

When researchers exogenously exposed sea urchin embryos to PAF, however, those embryos exhibited lower levels of caspase-3 and significantly more developed to the late-stage gastrula phase than the controls [35]. PAF-treated embryos were found to cleave caspase-3, suggesting that PAF may modulate apoptotic activity in the developing embryo through the intrinsic pathway.

While studies provide a correlation between PAF and apoptosis, and even the intrinsic pathway of apoptosis, in a developing embryo, few studies exist to date that look at how PAF, and 
furthermore, fluctuations in PAF, impact embryo viability as well as the mechanism that takes place.

\section{CONCLUSION}

Future work should aim at clarifying the mechanism that PAF uses to potentially regulate apoptosis. With the knowledge that PAF has a direct relationship with caspase-3 levels, further studies should focus on how caspase- 3 mediated activation of the apoptotic process affects early development.

This research can help us understand the basics on the role of apoptosis with the potential to increase the percentage of successful IVF procedures. Using sea urchins is a viable research model going forward due to the similarity to humans, and the cost/ time they take to use. There has been growing interest in using PAF as a therapeutic target; however, there have been few studies on the risk/benefits of using PAF to influence IVF success rate.

\section{REFERENCES}

1. Benveniste J, Henson PM, Cochrane CG (1972) Leukocyte-dependent histamine release from rabbit platelets: the role of IgE, basophils, and a platelet-activating factor. J Exp Med 136(6): 1356-1377.

2. Lordan R, Tsoupras A, Zabetakis I, Demopoulos CA (2019) Forty years since the structural elucidation of platelet-activating factor (PAF): Historical, current and future research perspectives. Molecules 24(23): 4414

3. Serhan CN, Chiang N, Dalli J, Levy BD (2015) Lipid mediators in the resolution of inflammation. Cold Spring Harbor perspectives in biology 7(2): a016311.

4. Grassi S, Francescangeli E, Goracci G, Pettorossi VE (1998) Role of platelet-activating factor in long-term potentiation of the rat medial vestibular nuclei. J Neurophysiology 79(6): 3266-3271.

5. Sahu RP (2015) Expression of the platelet-activating factor receptor enhances benzyl isothiocyanate-induced apoptosis in murine and human melanoma cells. Molec Med Reports 12(1): 394-400.

6. Roudebush WE, Massey JB, Elsner CW, Shapiro DB, Mitchell-Leef D, et al. (2005) The significance of platelet-activating factor and fertility in the male primate: A review. J Med Primatology 34(1): 20-24.

7. Tsoupras AB, Iatrou C, Frangia C, Demopoulos CA (2009) The implication of platelet activating factor in cancer growth and metastasis: Potent beneficial role of PAF-inhibitors and antioxidants. Infect Disord-Drug Targets 9(4): 390-399.

8. Feuerstein G, Rabinovici R, Leor J, Winkles JD, Vonhof S (1997) Plateletactivating factor and cardiac diseases: therapeutic potential for PAF inhibitors. J Lipid Mediat Cell Signal 15(3): 255-284.

9. López-Novoa JM (1999) Potential role of platelet activating factor in acute renal failure. Kidney Int 55(5): 1672-1682.

10. Roudebush WE, Purnell ET, Stoddart NR, Fleming SD (2002) Embryonic platelet-activating factor: temporal expression of the ligand and receptor. J Assist Reprod Genet 19(2): 72-78.

11. O'neill C (1995) Activity of platelet-activating factor acetylhydrolase in the mouse uterus during the estrous cycle, throughout the preimplantation phase of pregnancy and throughout the luteal phase of pseudopregnancy. Biol Reprod 52(5): 965-971.

12. Collier M, O’Neill C, Ammit AJ, Saunders DM (1988) Biochemical and pharmacological characterization of human embryo-derived platelet activating factor. Hum Reprod 3(8): 993-998.

13. Battye KM, Ammit AJ, O’Neill C, Evans G (1991) Production of plateletactivating factor by the pre-implantation sheep embryo. Reproduction 93(2): 507-514.

14. Mook JL, Diehl JR, Ashworth CJ, Mathur RS, Roudebush WE (1998) Presence of platelet-activating factor in porcine spermatozoa and uterine fluid. Theriogenology 1(49): 351.
15. Roudebush WE, Mathur S, Butler WJ (1994) Anti-platelet activating factor (PAF) antibody inhibits CFW mouse preimplantation embryo development. J Assist Reprod Genet 11(8): 414-418.

16. Spinks NR, O'Neill C (1988) Antagonists of embryo-derived plateletactivating factor prevent implantation of mouse embryos. Reproduction 84(1): 89-98.

17. Ando M, Suginami H, Matsuura S (1990) Pregnancy suppression by a structurally related antagonist for platelet activating factor CV-6209 in mice. Asia Oceania J Obstet Gynaecol 16(3): 283-290.

18. Roberts C, O’Neill C, Wright L (1993) Platelet activating factor (PAF) enhances mitosis in preimplantation mouse embryos. Reproduction, Fertility and Development 5(3): 271-279.

19. Ryan JP, Spinks NR, O’Neill C, Saunders DM, Wales RG (1987) Enhanced rates of implantation and increased metabolism of mouse embryos in the presence of platelet-activating factor. In Proc Fertil Soc Aust 21(8): 297-300.

20. Roudebush WE, Massey JB, Kort HI, Toledo AA, Mitchell-Leef D, et al. (2003) Exposure of preimplantation stage embryos to platelet-activating factor increases birth rate. Fertil Steril 21(8): 297-300.

21. Ryan JP, Spinks NR, O'Neill C, Ammit AJ, Wales RG (1989) Platelet activating factor (PAF) production by mouse embryos in vitro and its effect on embryonic metabolism. J Cellular Biochemistry 40(3): 387395.

22. Ryan JP, O’Neill C, Wales RG (1990) PAF directly influences the oxidative metabolism of glucose and lactate in the preimplantation mouse embryo. J Reprod Fertil 89: 301-307

23. Roudebush WE, Fukuda AI, Minhas BS (1993) Enhanced embryo development of rabbit oocytes fertilized in vitro with platelet activating factor (PAF)-treated spermatozoa. J Assist Reprod Genet 10(1): 91-94.

24. O'Neill C (2005) The role of PAF in embryo physiology. Human Reproduction Update 11(3): 215-228.

25. Ryan JP, O’Neill C, Ammit AJ, Roberts CG (1992) Metabolic and developmental responses of preimplantation embryos to platelet activating factor (PAF). Reprod Fertility Dev 4(4): 387-398.

26. Nishi O, Tominaga T, Goto Y, Hayashi K, Mori T (1995) Effects of platelet activating factor on mouse embryo implantation in vitro. J Assist Reprod Genet 12(5): 330-334.

27. Kölle S, Hughes B, Steele H (2020) Early embryo-maternal communication in the oviduct: A review. Molecular Reproduction and Development 87(6): 650-662.

28. Nakatsuka M, Yoshida N, Kudo T (1992) Platelet activating factor in culture media as an indicator of human embryonic development after in vitro fertilization. Human Reproduction 7(10): 1435-1439.

29. Amir Y, Insler M, Giller A, Gutman D, Atzmon G (2020) Senescence and longevity of sea urchins. Genes 11(5): 573.

30. Berdyshev EV, Vaskovsky VE, Vaschenko MA (1995) Sea urchins-a new model for PAF research in embryology. Comp Biochem Physiol B Biochem Mol Biol 110(3): 629-632.

31. Oyugi T, Grasso LM, Leblang C, Tyler L, Lal A, et al. (2019) Regulation of embryonic development by platelet-activating factor is most likely via the intrinsic apoptosis pathway. Fertil Steril 112(3): e74.

32. Roudebush WE, Toledo AA, Kort HI, Mitchell-Leef D, Elsner CW, et al. (2004) Platelet-activating factor significantly enhances intrauterine insemination pregnancy rates in non-male factor infertility. Fertil Steril 82(1): 52-56

33. Lal A, Blalock J, Chosed RJ, Roudebush WE (2019) Platelet activating factor reduces Caspase-3 activity and enhances embryo development in the sea urchin (Lytechinus variegatus). Biomed.

34. Ryan SD, Harris CS, Mo F, Lee H, Hou ST, et al. (2007) Platelet activating factor-induced neuronal apoptosis is initiated independently of its Gprotein coupled PAF receptor and is inhibited by the benzoate orsellinic acid. J Neurochem 103(1): 88-97. 
35. Ma X, Bazan HE (2001) Platelet-activating factor (PAF) enhances apoptosis induced by ultraviolet radiation in corneal epithelial cells through cytochrome c-caspase activation. Current Eye Research 23(5): 326-335.

36. Thurber RV, Epel D (2007) Apoptosis in early development of the sea urchin, Strongylocentrotus purpuratus. Dev Biol 303(1): 336-346.

37. Angermayer ME, Chosed RJ, Roudebush WE (2018) Platelet-activating factor protects early embryos from apoptosis. Reproductive Sciences 25(S1): 245A.
38. Ouygi T, Grasso LM, Leblang C, Tyler L, Lal A, et al. (2019) Regulation of embryonic development by platelet-activating factor is most likely via the intrinsic apoptosis pathway. Fertil Steril 112(3): e74.

39. Carter AD, Sible JC (2003) Loss of XChk1 function triggers apoptosis after the midblastula transition in Xenopus laevis embryos. Mech Dev 120(3): 315-323.

40. Lesser MP, Kruse VA, Barry TM (2003) Exposure to ultraviolet radiation causes apoptosis in developing sea urchin embryos. J Exp Biol 206(22): 4097-4103. 